\title{
On the estimates in various spaces to the control function of the extremum problem for parabolic equation
}

\author{
IRINA ASTASHOVA \\ Lomonosov Moscow State University \\ Department of Mathematics and Mechanics \\ Leninskie Gory, 1, 119991, Moscow \\ Plekhanov Russian University of Economics \\ Stremyanny lane, 36, 117997, Moscow \\ RUSSIAN FEDERATION
}

\author{
ALEXEY FILINOVSKIY \\ Bauman Moscow State Technical University \\ 2 nd Baumanskaya str., 5, 105005, Moscow \\ Lomonosov Moscow State University \\ Department of Mathematics and Mechanics \\ Leninskie Gory, 1, 119991, Moscow \\ RUSSIAN FEDERATION
}

\author{
DMITRIY LASHIN \\ FITO Research and Production Company \\ Kaluzhskoe shosse, 23 km, 14-3, Moscow \\ RUSSIAN FEDERATION
}

\begin{abstract}
For the minimization problem with pointwise observation governed by a one-dimensional parabolic equation with a free convection term and a depletion potential, we formulate a result on the existence and uniqueness of a minimizer from a prescribed set. We use a weight quadratic cost functional showing the temperature deviation. We obtain estimates for the norm of control functions in terms of the value of the quality functional in different functional spaces. It gives us a possibility to estimate the required internal energy of the system. To prove theseresultsweestablishthepositivityprinciple.
\end{abstract}

Key-Words: Parabolic equation, pointwise observation, extremum problem, control function, estimates.

Received: February 12, 2021. Revised: September 4, 2021. Accepted: September 13, 2021. Published: October 1, 2021.

\section{Introduction}

We consider the extremum problem with weighted integral cost functional for the following parabolic mixed problem

$$
\begin{aligned}
& u_{t}=\left(a(x, t) u_{x}\right)_{x}+b(x, t) u_{x}+d(x, t) u, \\
& (x, t) \in Q_{T}=(0,1) \times(0, T), \quad T>0, \\
& u(0, t)=\varphi(t), \quad u_{x}(1, t)=\psi(t), \\
& 0<t<T, \\
& u(x, 0)=\xi(x), \quad 0<x<1,
\end{aligned}
$$

where the real functions $a, b$ and $d$ are smooth in $\bar{Q}_{T}$,

$$
0<a_{0} \leq a(x, t) \leq a_{1}<\infty
$$

$\varphi \in W_{2}^{1}(0, T), \psi \in W_{2}^{1}(0, T), \xi \in L_{2}(0,1)$. Here $W_{2}^{1}(0, T)$ is Sobolev space of weakly differentiable functions with the norm

$$
\|u\|_{W_{2}^{1}(0, T)}=\left(\int_{0}^{T}\left(\left|u^{\prime}\right|^{2}+|u|^{2}\right) d t\right)^{1 / 2} .
$$

We study the control problem with a point observation: by controlling the temperature $\varphi$ at the left end of the segment (the functions $\psi$ and $\xi$ are assumed to be fixed), we try to make at some point $x_{0} \in(0,1)$ the temperature $u\left(x_{0}, t\right)$ close to the given function $z(t)$ over the entire time interval $(0, T)$.

This problem arises in the model of climate control in industrial greenhouses [1] - [2].

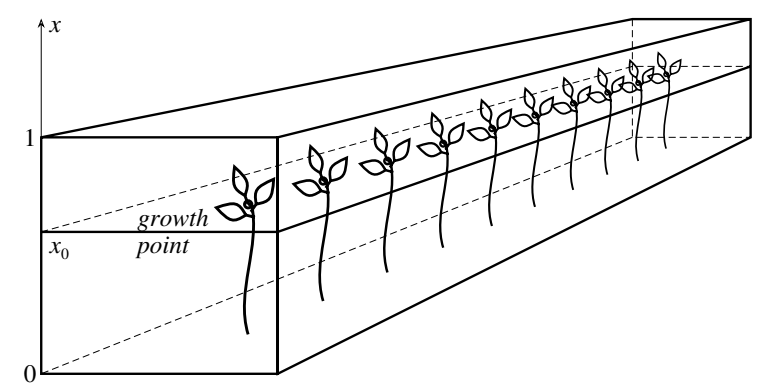

Note that extremal problems for parabolic equations were considered in [10] - [13] (as usual, problems with final or distributed observation). But the results and methods of investigation are not similar to our methods.

The proposed paper develops and generalizes the authors' results of [1] - [8]. We study here a more 
general equation with a variable diffusion coefficient $a$, a convection coefficient $b$, and a potential $d$, called the depletion potential, and obtain lower estimates of control function $\varphi$ with the help of the value of a quality functional in various spaces.

Some methods of study the parabolic control problems is used in [14], but there were no results similar to presented in our article (for example, estimates of control functions or positivity principle).

Definition 1. ([9], p. 6.) We denote by $V_{2}^{1,0}\left(Q_{T}\right)$ the Banach space of functions $u \in W_{2}^{1,0}\left(Q_{T}\right)$ with the finite norm

$$
\begin{aligned}
& \|u\|_{V_{2}^{1,0}\left(Q_{T}\right)} \\
& =\sup _{0 \leq t \leq T}\|u(x, t)\|_{L_{2}(0,1)}+\left\|u_{x}\right\|_{L_{2}\left(Q_{T}\right)}
\end{aligned}
$$

such that $t \mapsto u(\cdot, t)$ is a continuous mapping $[0, T] \rightarrow L_{2}(0,1)$

We denote by $\widetilde{W}_{2}^{1}\left(Q_{T}\right)$ the set of functions $\eta \in$ $W_{2}^{1}\left(Q_{T}\right)$ satisfying the conditions $\eta(x, T)=0$, $\eta(0, t)=0$.

Definition 2. A weak solution to problem (1) - (3) is a function $u \in V_{2}^{1,0}\left(Q_{T}\right)$ satisfying the condition $u(0, t)=\varphi(t)$ and the equality

$$
\begin{aligned}
& \int_{Q_{T}}\left(a(x, t) u_{x} \eta_{x}-b(x, t) u_{x} \eta\right. \\
& \left.-d(x, t) u \eta-u \eta_{t}\right) d x d t \\
& =\int_{0}^{1} \xi(x) \eta(x, 0) d x \\
& +\int_{0}^{T} a(1, t) \psi(t) \eta(1, t) d t
\end{aligned}
$$

for all $\eta \in \widetilde{W}_{2}^{1}\left(Q_{T}\right)$.

\section{Main Results}

Theorem 3. ([7] - [8]) The problem (1) - (3) has a unique weak solution $u \in V_{2}^{1,0}\left(Q_{T}\right)$, and it satisfies the inequality

$$
\begin{aligned}
& \|u\|_{V_{2}^{1,0}\left(Q_{T}\right)} \\
& \leq C_{1}\left(\|\varphi\|_{W_{2}^{1}(0, T)}+\|\psi\|_{W_{2}^{1}(0, T)}+\|\xi\|_{L_{2}(0,1)}\right),
\end{aligned}
$$

with some constant $C_{1}$ independent of $\varphi, \psi$ and $\xi$.

Corollary 4. The solution $u$ of the problem (1) (3) continuously depends on the initial datum $\xi$ and boundary data $\varphi$, $\psi$ from $L_{2}(0,1) \times W_{2}^{1}(0, T) \times$ $W_{2}^{1}(0, T)$ to $V_{2}^{1,0}\left(Q_{T}\right)$.
Note that a numerical solution of a parabolic Dirichlet boundary value problem is obtained in [15].

To obtain the next estimate we need the following positivity principle.

Theorem 5. Let $u$ be a solution of the problem (1) - (3) with the boundary and initial functions $\varphi, \psi$ and $\xi$ satisfying the conditions ess $\inf _{t \in(0, T)} \varphi \geq 0$, ess $\inf _{t \in(0, T)} \psi \geq 0, t \in[0, T]$, ess $\inf _{t \in(0,1)} \xi \geq 0, x \in$ $[0,1]$. Then the solution $u$ is nonegative, too:

$$
\text { ess } \inf _{(x, t) \in Q_{T}} u \geq 0 .
$$

Using Theorem 5, in the case of nonnegative $\varphi, \psi$ and $\xi$, we have the following estimate.

Theorem 6. Let the functions $a, b, d$ satisfy the conditions $a_{t} \geq 0, b_{x}-d \geq 0,(x, t) \in Q_{T} ; b \geq 0$, $(x, t) \in\left[0, x_{0}\right] \times[0, T], x_{0} \in(0,1] ; b(1, t) \leq 0$, $t \in[0, T] ; \varphi \geq 0, \psi \geq 0, \xi \geq 0$. Then for the solution $u$ of the problem (1) - (3) the inequality

$$
\begin{aligned}
& \left\|u\left(x_{0}, t\right)\right\|_{L_{1}(0, T)} \leq\|\varphi\|_{L_{1}(0, T)} \\
& +\frac{x_{0}}{a_{1}}\left(a_{2}\|\psi\|_{L_{1}(0, T)}+\|\xi\|_{L_{1}(0,1)}\right),
\end{aligned}
$$

holds.

Corollary 7. Let $a, b, d$ satisfy the conditions of Theorem $6, \varphi \geq 0, \psi=0, \xi=0$. Then, for solution of the problem (1)-(3), the following inequality holds:

$$
\left\|u\left(x_{0}, t\right)\right\|_{L_{1}(0, T)} \leq\|\varphi\|_{L_{1}(0, T)} .
$$

We denote by $\Phi \subset W_{2}^{1}(0, T)$ the set of control functions $\varphi$ and by $Z \subset L_{2}(0, T)$ the set of objective functions $z$. We further suppose that $\Phi$ is nonempty, closed, convex and bounded set. Consider the weighted integral cost functional

$$
\begin{aligned}
& J[z, \rho, \varphi]=\int_{0}^{T}\left|u_{\varphi}\left(x_{0}, t\right)-z(t)\right|^{2} \rho(t) d t, \\
& x_{0} \in(0,1), \quad \varphi \in \Phi, \quad z \in Z,
\end{aligned}
$$

where $u_{\varphi} \in V_{2}^{1,0}\left(Q_{T}\right)$ is the solution to the problem (1) - (3) with the given control function $\varphi$. Here $\rho \in L_{\infty}(0, T)$ is a real-valued weight function such that

$$
\begin{aligned}
0<\rho_{1} & =\text { ess } \inf _{t \in(0, T)} \rho(t), \\
\rho_{2} & =\text { ess } \sup _{t \in(0, T)} \rho(t) .
\end{aligned}
$$

Assuming the functions $z$ and $\rho$ to be fixed, consider the minimization problem

$$
m[z, \rho, \Phi]=\inf _{\varphi \in \Phi} J[z, \rho, \varphi] .
$$




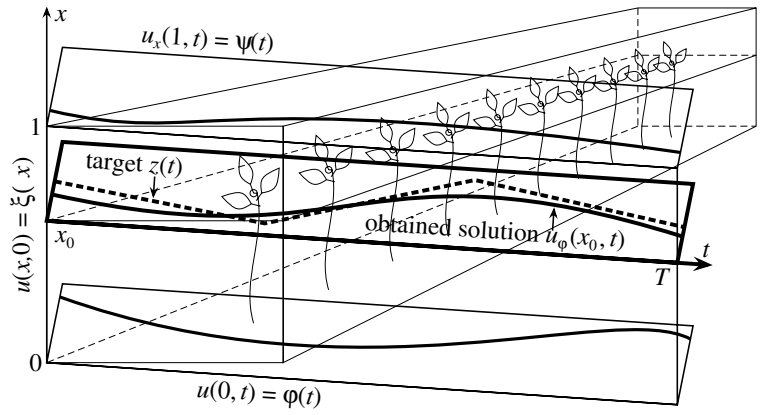

In ([7] - [8]) the following result is obtained.

Theorem 8. For any $z \in L_{2}(0, T)$ there exists a unique function $\varphi_{0} \in \Phi$ such that

$$
m[z, \rho, \Phi]=J\left[z, \rho, \varphi_{0}\right] .
$$

Theorem 6 implies a lower estimate for the norm of control functions in terms of the value of the quality functional, as you can see from the following theorem.

Theorem 9. Let $x_{0}$ and the functions $a, b, d, \varphi, \psi, \xi$ satisfy the conditions of Theorem 6. Then the following inequality holds:

$$
\begin{aligned}
& \|\varphi\|_{L_{1}(0, T)} \\
& \geq \max \left\{0,\|z\|_{L_{1}(0, T)}-\left(\frac{T J[\varphi, \rho, z]}{\rho_{1}}\right)^{1 / 2}\right. \\
& \left.-\frac{x_{0}}{a_{1}}\left(a_{2}\|\psi\|_{L_{1}(0, T)}+\|\xi\|_{L_{1}(0,1)}\right)\right\} .
\end{aligned}
$$

The resulting estimate (16) allows us to obtain information on control functions. It is useful for estimating the internal energy of the system necessary to achieve a required value of the functional $J$.

Corollary 10. Let $x_{0}$ and the functions $a, b, d, \varphi$ satisfy the conditions of Theorem 6. Suppose $\psi=\xi=0$. Then the following inequality holds:

$$
\begin{aligned}
& \|\varphi\|_{L_{1}(0, T)} \\
& \geq \max \left\{0,\|z\|_{L_{1}(0, T)}-\left(\frac{T J[\varphi, \rho, z]}{\rho_{1}}\right)^{1 / 2}\right\} .
\end{aligned}
$$

We can also give lower estimates to the various norms of the control function $\varphi$.

Theorem 11. Let $x_{0}$ and the functions $a, b, d, \varphi, \psi, \xi$ satisfy the conditions of Theorem 6. Then the following inequality holds:

$$
\begin{aligned}
& \|\varphi\|_{L_{2}(0, T)} \geq \max \left\{0, \frac{1}{T^{1 / 2}}\|z\|_{L_{1}(0, T)}\right. \\
& -\left(\frac{J[\varphi, \rho, z]}{\rho_{1}}\right)^{1 / 2} \\
& \left.-\frac{x_{0}}{a_{1} T^{1 / 2}}\left(a_{2}\|\psi\|_{L_{1}(0, T)}+\|\xi\|_{L_{1}(0,1)}\right)\right\} .
\end{aligned}
$$

Theorem 12. Let $x_{0} \in(0,1]$ and the functions $a, b, d$, $\varphi, \psi$ and $\xi$ satisfy the conditions of Theorem 6. Then the following inequality holds:

$$
\begin{aligned}
& \|\varphi\|_{W_{2}^{1}(0, T)} \geq \max \left\{0, \frac{1}{T^{1 / 2}}\|z\|_{L_{1}(0, T)}\right. \\
& -\left(\frac{J[\varphi, \rho, z]}{\rho_{1}}\right)^{1 / 2} \\
& \left.-\frac{x_{0}}{a_{1} T^{1 / 2}}\left(a_{2}\|\psi\|_{L_{1}(0, T)}+\|\xi\|_{L_{1}(0,1)}\right)\right\} .
\end{aligned}
$$

\section{Proofs}

Proof of theorem 6. At first, let us suppose, that the boundary and initial data of the problem (1) - (3) are smooth and the solution $u$ is smooth, too. Integrating equation (1) by $x$ over the interval $(z, 1), z \in(0,1)$ and using the boundary condition (2), we obtain the following equality:

$$
\begin{aligned}
& \int_{z}^{1} u_{t}(x, t) d x+a(z, t) u_{x}(z, t)-a(1, t) \psi(t) \\
& -b(1, t) u(1, t)+b(z, t) u(z, t) \\
& +\int_{z}^{1}\left(b_{x}(x, t)-d(x, t)\right) u(x, t) d x .
\end{aligned}
$$

Now, we divide the equality (20) by the positive function $a(z, t)$ and integrate over the integral $\left(0, x_{0}\right)$, where $x_{0} \in(0,1)$ is a given point. Therefore,

$$
\begin{aligned}
& \int_{0}^{x_{0}} \frac{d z}{a(z, t)} \int_{z}^{1} u_{t}(x, t) d x+u\left(x_{0}, t\right)-\varphi(t) \\
& -a(1, t) \psi(t) \int_{0}^{x_{0}} \frac{d z}{a(z, t)} \\
& -b(1, t) u(1, t) \int_{0}^{x_{0}} \frac{d z}{a(z, t)}+\int_{0}^{x_{0}} \frac{b(z, t) u(z, t) d z}{a(z, t)} \\
& +\int_{0}^{x_{0}} \frac{d z}{a(z, t)} \int_{z}^{1}\left(b_{x}(x, t)-d(x, t)\right) u(x, t) d x
\end{aligned}
$$

The next step of proof is the integration of the resulting equality (21) by $t$ over the interval $(0, T)$. We 
obtain

$$
\begin{aligned}
& \int_{0}^{T} d t \int_{0}^{x_{0}} \frac{d z}{a(z, t)} \int_{z}^{1} u_{t}(x, t) d x \\
& +\int_{0}^{T} u\left(x_{0}, t\right) d t-\int_{0}^{T} \varphi(t) d t \\
& -\int_{0}^{T} a(1, t) \psi(t) d t \int_{0}^{x_{0}} \frac{d z}{a(z, t)} \\
& -\int_{0}^{T} b(1, t) u(1, t) d t \int_{0}^{x_{0}} \frac{d z}{a(z, t)} \\
& +\int_{0}^{T} d t \int_{0}^{x_{0}} \frac{b(z, t) u(z, t) d z}{a(z, t)} \\
& +\int_{0}^{T} d t \int_{0}^{x_{0}} \frac{d z}{a(z, t)} \times \\
& \times \int_{z}^{1}\left(b_{x}(x, t)-d(x, t)\right) u(x, t) d x .
\end{aligned}
$$

Integration by parts of (22) gives us the following relation

$$
\begin{aligned}
& \int_{0}^{T} u\left(x_{0}, t\right) d t+\int_{0}^{x_{0}} \frac{d z}{a(z, T)} \int_{z}^{1} u(x, T) d x \\
& +\int_{0}^{T} d t \int_{0}^{x_{0}} \frac{a_{t}(z, t)}{a^{2}(z, t)} d z \int_{z}^{1} u(x, t) d x \\
& -\int_{0}^{T} b(1, t) u(1, t) d t \int_{0}^{x_{0}} \frac{d z}{a(z, t)} \\
& +\int_{0}^{T} d t \int_{0}^{x_{0}} \frac{b(z, t) u(z, t) d z}{a(z, t)} \\
& +\int_{0}^{T} d t \int_{0}^{x_{0}} \frac{d z}{a(z, t)} \times \\
& \times \int_{z}^{1}\left(b_{x}(x, t)-d(x, t)\right) u(x, t) d x \\
& \leq \int_{0}^{T} \varphi(t) d t+\int_{0}^{T} a(1, t) \psi(t) d t \int_{0}^{x_{0}} \frac{d z}{a(z, t)} \\
& +\int_{0}^{x_{0}} \frac{d z}{a(z, 0)} \int_{z}^{1} \xi(x) d x .
\end{aligned}
$$

Using Theorem 5, we obtain the non-negativity of the function $u$ in $\bar{Q}_{T}$. Therefore, from equality (23), using the conditions $a_{t} \geq 0, b_{x}-d \geq 0,(x, t) \in Q_{T} ; b \geq 0$, $(x, t) \in\left[0, x_{0}\right] \times[0, T], x_{0} \in(0,1] ; b(1, t) \leq 0$, $t \in[0, T] ; \varphi \geq 0, \psi \geq 0, \xi \geq 0$ and the inequality $u \geq 0$, we get the following inequality:

$$
\begin{aligned}
& \int_{0}^{T} u\left(x_{0}, t\right) d t \\
& \leq \int_{0}^{T} \varphi(t) d t+\int_{0}^{T} a(1, t) \psi(t) d t \int_{0}^{x_{0}} \frac{d z}{a(z, t)} \\
& +\int_{0}^{x_{0}} \frac{d z}{a(z, 0)} \int_{z}^{1} \xi(x) d x
\end{aligned}
$$

Combining (4) and (24), we obtain

$$
\begin{aligned}
& \int_{0}^{T} u\left(x_{0}, t\right) d t \\
& \leq \int_{0}^{T} \varphi(t) d t+\int_{0}^{T} a_{2} \psi(t) d t \int_{0}^{x_{0}} \frac{d z}{a_{1}} \\
& +\int_{0}^{x_{0}} \frac{d z}{a_{1}} \int_{z}^{1} \xi(x) d x \\
& \leq \int_{0}^{T} \varphi(t) d t \\
& +\frac{a_{2} x_{0}}{a_{1}} \int_{0}^{T} \psi(t) d t+\frac{x_{0}}{a_{1}} \int_{0}^{1} \xi(x) d x .
\end{aligned}
$$

For non-negative functions, inequality (25) implies the inequality

$$
\begin{aligned}
& \left\|u\left(x_{0}, t\right)\right\|_{L_{1}(0, T)} \leq\|\varphi\|_{L_{1}(0, T)} \\
& +\frac{x_{0}}{a_{1}}\left(a_{2}\|\psi\|_{L_{1}(0, T)}+\|\xi\|_{L_{1}(0,1)}\right) .
\end{aligned}
$$

Hence, we prove (10) for smooth data and smooth solutions. To prove it for all prescribed boundary and initial functions, we have to take the sequences of smooth functions $\varphi_{j}, \psi_{j}$ and $\xi_{j}$ such that $\left\|\varphi_{j}\right\|_{W_{2}^{1}(0, T)} \rightarrow 0,\left\|\psi_{j}\right\|_{W_{2}^{1}(0, T)} \rightarrow 0$, and $\left\|\xi_{j}\right\|_{L_{2}(0,1)} \rightarrow 0, j \rightarrow \infty$. Now, we have the estimate (10) for the corresponding solutions $u_{j}$, and obtain, by the inequality (8), the estimate (10) for the limit function $u$. Proof of Theorem 5 is complete.

For further considerations we need the following elementary inequality.

Lemma 13. Let $B$ be a Banach space with the norm $\|\cdot\|_{B}$. Then for all $g \in B, h \in B$ we have

$$
\|g\|_{B} \geq \max \left\{0,\|h\|_{B}-\|h-g\|_{B}\right\} .
$$

Proof of lemma 13. The application of the triangle inequality $\|p+q\|_{B} \leq\|p\|_{B}+\|q\|_{B}$ with $p=g$, $q=h-g$ yields

$$
\|h\|_{B} \leq\|g\|_{B}+\|h-g\|_{B} .
$$

Combining (27) and the relation $\|g\| \geq 0$, we obtain the inequality (26).

Proof of theorem 9. Applying lemma 13 for $B=$ $L_{1}(0, T), g=u\left(x_{0}, t\right), h=z(t)$, we get

$$
\begin{aligned}
& \left\|u_{\varphi}\left(x_{0}, \cdot\right)\right\|_{L_{1}(0, T)} \\
& \geq \max \left\{0,\|z(\cdot)\|_{L_{1}(0, T)}\right. \\
& \left.-\left\|u_{\varphi}\left(x_{0}, \cdot\right)-z(\cdot)\right\|_{L_{1}(0, T)}\right\} .
\end{aligned}
$$


Now, It follows from Holder inequality and (13) that

$$
\begin{aligned}
& \left\|u_{\varphi}\left(x_{0}, \cdot\right)-z(\cdot)\right\|_{L_{1}(0, T)} \\
& \leq T^{1 / 2}\left\|u_{\varphi}\left(x_{0}, \cdot\right)-z(\cdot)\right\|_{L_{2}(0, T)} \\
& \leq\left(\frac{T J[\varphi, \rho, z]}{\rho_{1}}\right)^{1 / 2}
\end{aligned}
$$

By (10) and (29) we obtain

$$
\begin{aligned}
& \|\varphi\|_{L_{1}(0, T)} \geq\left\|u_{\varphi}\left(x_{0}, \cdot\right)\right\|_{L_{1}(0, T)} \\
& -\frac{x_{0}}{a_{1}}\left(a_{2}\|\psi\|_{L_{1}(0, T)}+\|\xi\|_{L_{1}(0,1)}\right) \\
& \geq\|z(t)\|_{L_{1}(0, T)}-\left(\frac{T J[\varphi, \rho, z]}{\rho_{1}}\right)^{1 / 2} \\
& -\frac{x_{0}}{a_{1}}\left(a_{2}\|\psi\|_{L_{1}(0, T)}+\|\xi\|_{L_{1}(0,1)}\right) .
\end{aligned}
$$

Proof of theorem 11. By Holder inequality we have

$$
\|\varphi\|_{L_{1}(0, T)} \leq T^{1 / 2}\|\varphi\|_{L_{2}(0, T)} .
$$

Combining (16) and (31) we get the estimate

$$
\begin{aligned}
& T^{1 / 2}\|\varphi\|_{L_{2}(0, T)} \geq \max \left\{0,\|z\|_{L_{1}(0, T)}\right. \\
& -\left(\frac{T^{1 / 2} J[\varphi, \rho, z]}{\rho_{1}}\right)^{1 / 2} \\
& \left.-\frac{x_{0}}{a_{1}}\left(a_{2}\|\psi\|_{L_{1}(0, T)}+\|\xi\|_{L_{1}(0,1)}\right)\right\} .
\end{aligned}
$$

This completes the proof of theorem 11 .

Proof of theorem 12. By (5) we have

$$
\begin{aligned}
& \|\varphi\|_{W_{2}^{1}(0, T)}^{2}=\left\|\varphi^{\prime}\right\|_{L_{2}(0, T)}^{2} \\
& +\|\varphi\|_{L_{2}(0, T)}^{2} \geq\|\varphi\|_{L_{2}(0, T)}^{2} .
\end{aligned}
$$

so

$$
\|\varphi\|_{W_{2}^{1}(0, T)} \geq\|\varphi\|_{L_{2}(0, T)} .
$$

Now, by (29) we obtain

$$
\begin{aligned}
& \|\varphi\|_{L_{2}(0, T)} \geq \max \left\{0, \frac{1}{T^{1 / 2}}\|z\|_{L_{1}(0, T)}\right. \\
& -\left(\frac{J[\varphi, \rho, z]}{\rho_{1}}\right)^{1 / 2} \\
& \left.-\frac{x_{0}}{a_{1} T^{1 / 2}}\left(a_{2}\|\psi\|_{L_{1}(0, T)}+\|\xi\|_{L_{1}(0,1)}\right)\right\} .
\end{aligned}
$$

So, from (33) and (34) it follows that

$$
\begin{aligned}
& \|\varphi\|_{W_{2}^{1}(0, T)} \geq \max \left\{0, \frac{1}{T^{1 / 2}}\|z\|_{L_{1}(0, T)}\right. \\
& -\left(\frac{J[\varphi, \rho, z]}{\rho_{1}}\right)^{1 / 2} \\
& \left.-\frac{x_{0}}{a_{1} T^{1 / 2}}\left(a_{2}\|\psi\|_{L_{1}(0, T)}+\|\xi\|_{L_{1}(0,1)}\right)\right\} .
\end{aligned}
$$

\section{Open problems}

1. It is interesting to obtain upper estimates in different spaces for control function $\varphi$ similar to lower estimates (16), (18), (19).

2. An important problem is to prove that $m\left[z, \rho, W_{2}^{1}(0, T)\right]=0$ for any $z \in L_{2}(0, T)$ and $\rho \in L_{\infty}(0, T)$ to the equation (1) with general type variable coefficients $a(x, t), b(x, t), d(x, t)$. Now this result is proved for equations with coefficients independent of $t$ (see $[4,5,6]$ ).

Acknowledgement. The research was partially supported by Russian Science Foundation (scientific project 20-11-20272).

\section{References:}

[1] Astashova I. V., Filinovskiy A. V., Lashin D. A. On maintaining optimal temperatures in greenhouses. WSEAS Trans. on Circuits and Systems, 15, no. 23, (2016), $198-204$.

[2] Astashova I., Filinovskiy A., Lashin D. On optimal temperature control in hothouses. Proc. Int. Conf. on Numerical Analysis and Applied Mathematics 2016, AIP Conf. Proc., 2017, 4 - 8.

[3] Astashova I. V., Filinovskiy A. V. On the controllability in parabolic problem with time distributed functional. Differ. equ., 53, (2018), 851 -853 .

[4] Astashova I. V., Filinovskiy A. V. On the dense controllability for the parabolic problem with time-distributed functional. Tatra Mt. Math. Publ., 71, (2018), 9 - 25.

[5] Astashova I. V., Filinovskiy A. V. On properties of minimizers of a control problem with timedistributed functional related to parabolic equations. Opuscula Math., 39, (2019), 595 - 609. 
[6] Astashova I. V., Lashin D. A., Filinovskiy A. V. Control with point observation for a parabolic problem with convection. Trans. Moscow Math. Soc., 80 (2019), 221 - 234.

[7] Astashova I. V., Filinovskiy A. V. Controllability and Exact Controllability in a Problem of Heat Transfer with Convection and Time Distributed Functional. J. Math. Sci., 244, (2020), 148 157.

[8] Astashova I. V., Lashin D. A., Filinovskiy A. V. On a control problem with point observation for a parabolic equation in the presence of convection and depletion potential. Differ. Equ., 56, (2020), 828 - 829 .

[9] Ladyzhenskaya O. A. , Solonnikov V. A., Ural'seva N. N., Linear and quasi-linear equations of parabolic type. Translations of Mathematical Monographs, 23, American Mathematical Society, Providence, RI, 1968.

[10] Troltzsch F. Optimal Control of Partial Differential Equations. Theory, Methods and Applications. Graduate Studies in Mathematics, 112, Providence, AMS, 2010.

[11] Lurie K. A. Applied Optimal Control Theory of Distributed Systems. Springer, Berlin, 2013.

[12] V. Dhamo, F. Tröltzsch, Some aspects of reachability for parabolic boundary control problems with control constraints. Comput Optim Appl (2011) 50:75-110.

[13] Şener, Ş.S., Subaşi, M. On a Neumann boundary control in a parabolic system. Bound Value Probl 2015, 166 (2015).

[14] J.L. Lions, Optimal Control of Systems governed by Partial Differential Equations, Springer, Berlin, 1971.

[15] Burova I. G., Muzafarova E. F., Approximations with Polynomial, Trigonometric, Exponential Splines of the Third Order and Boundary Value Problem. Int. J. of Circuits, Systems and Signal Processing, 14, (2020), 460 - 473.

\section{Creative Commons Attribution License 4.0 (Attribution 4.0 International, CC BY 4.0)}

This article is published under the terms of the Creative Commons Attribution License 4.0

https://creativecommons.org/licenses/by/4.0/deed.en_US 